\title{
Kinetics and mechanism of the oxidation of some neutral and acidic $\alpha$-amino acids by tetrabutylammonium tribromide
}

\author{
RAGHVENDRA SHUKLA ${ }^{\mathrm{a}}$, PRADEEP K SHARMA ${ }^{\mathrm{a}}$ and KALYAN K BANERJI ${ }^{\mathrm{b}}$ * \\ ${ }^{a}$ Department of Chemistry, JNV University, Jodhpur 342 005, India \\ ${ }^{\mathrm{b}}$ Faculty of Science, National Law University, Mandore (NH 65), Jodhpur 342 304, India \\ e-mail: banerjikk@rediffmail.com
}

MS received 15 April 2003; revised 13 October 2003

\begin{abstract}
The oxidation of eleven amino acids by tetrabutylammonium tribromide (TBATB) in aqueous acetic acid results in the formation of the corresponding carbonyl compounds and ammonia. The reaction is first order with respect to TBATB. Michaelis-Menten type kinetics is observed with some of the amino acids while others exhibit second-order dependence. It failed to induce polymerization of acrylonitrile. The effect of solvent composition indicate that the rate of reaction increases with increase in the polarity of the medium. Addition of tetrabutylammonium chloride has no effect on the rate of oxidation. Addition of bromide ion causes decrease in the oxidation rate but only to a limiting value. The reaction is susceptible to both polar and steric effects of the substituents. A suitable mechanism has been proposed.
\end{abstract}

Keywords. Amino acid; tetraalkylammonium tribromide; kinetics; mechanism; oxidation.

\section{Introduction}

Tetraalkylammonium polyhalides are widely used as halogenating reagents in synthetic organic chemistry. ${ }^{1-3}$ Tetrabutylammonium tribromide (TBATB) has been used for the bromination of some selected organic substrates. ${ }^{4}$ There are, however, only a few reports regarding their use as oxidizing and brominating agents in synthetic chemistry. ${ }^{5-7}$ These compounds are more suitable than molecular halogens because of their solid nature, ease of handling, stability, selectivity and excellent product yields. We have been interested in the kinetic and mechanistic studies of the reactions of polyhalides and many reports including those on TBATB have already emanated from our laboratory. ${ }^{8-10}$ There seems to be no report on the oxidation of $\alpha$-amino acids by TBATB. A study of the kinetics of the oxidation of amino acids is important both from a mechanistic point of view and its bearing on the mechanism of amino acid metabolism. Therefore, we have studied the oxidation of several neutral and acidic $\alpha$-amino acids by TBATB in aqueous acetic acid solution, and the mechanistic aspects are discussed in this paper.

\footnotetext{
*For correspondence
}

\section{Experimental}

\subsection{Materials}

All the amino acids were commercial products of highest degree of purity and were used as supplied. Perdeuterioglycine $\left(\mathrm{ND}_{2} \mathrm{CD}_{2} \mathrm{COOD}\right)$ was obtained from Sigma Chemicals (USA). TBATB was prepared by the reported method ${ }^{1}$ and its purity was checked by an iodometric method. Acetic acid was refluxed with chromic oxide and acetic anhydride for $3 \mathrm{~h}$ and then fractionally distilling it. ${ }^{11}$ All other reagents were commercial products and were purified by the usual methods. ${ }^{11}$

\subsection{Product analysis}

The main products of the amino acids were the corresponding carbonyl compounds and ammonia. The presence of ammonia in the reaction mixture was detected by the test with $p$-nitrobenzenediazonium chloride. $^{12}$

In a typical experiment, $\alpha$-alanine $(4.45 \mathrm{~g}, 0.05 \mathrm{~mol})$ and TBATB (4.45 g, 0.01 mol) were made up to $100 \mathrm{ml}$ in $1: 1(v / v)$ acetic acid-water. The mixture was allowed to stand for $\approx 15 \mathrm{~h}$ in the dark to ensure 
completion of the reaction. It was then treated with an excess $(250 \mathrm{ml})$ of a saturated solution of 2,4dinitrophenylhydrazine in $2 \mathrm{~mol} \mathrm{dm}^{-3} \mathrm{HCl}$ and kept in a refrigerator for $\approx 10 \mathrm{~h}$. The precipitated 2,4dinitrophenylhydrazone (DNP) was filtered off, dried, weighed, recrystallized from ethanol and weighed again. The yields of DNP before and after recrystallization were $2.24 \mathrm{~g}(95 \%)$ and $1.97 \mathrm{~g}$ $(83 \%)$ respectively, based on the consumption of TBATB. The DNP was found to be identical (m.pt. and mixed m.pt.) with the DNP of acetaldehyde. In similar experiments, with other amino acids, the yields of the DNP were in the range of $80-89 \%$ after recrystallization.

Stoichiometry, with an excess of amino acid could not be studied because of the difficulty in determining the concentration of amino acids. Stoichiometric determination with an excess of TBATB showed that two moles of TBATB were consumed per mole of the amino acid. This is because aldehydes, the initial products of the oxidation, were further oxidized to carboxylic acids.

\subsection{Kinetic measurements}

The reactions were studied under pseudo-first-order conditions by keeping an excess $(\times 15$ or greater) of the substrate over TBATB. The solvent was $1: 1$ $(v / v)$ acetic acid-water, unless mentioned otherwise. Tribromide ion is known to dissociate to a large extent to bromine and bromide ion. The value ${ }^{13}$ of the dissociation constant in 1:1 $(\mathrm{v} / \mathrm{v})$ acetic acid-water is $\approx 0.02 \mathrm{~mol} \mathrm{dm}^{-3}$. To suppress the dissociation, all kinetic runs were carried out in the presence of an excess $\left(0.3 \mathrm{~mol} \mathrm{dm}^{-3}\right)$ of potassium bromide. The reactions were studied at constant temperature $( \pm 0.1 \mathrm{~K})$ and were followed by monitoring the decrease in the concentration of TBATB at $394 \mathrm{~nm}$ for up to $80 \%$ reaction. Pseudo-first-order rate constants, $k_{\text {obs }}$, were evaluated from linear plots $(r>0.995)$ of $\log$ [TBATB] against time. Duplicate kinetic runs showed that the rate constants are reproducible to within $\pm 3 \%$. Simple and multivariate regression analyses were carried out by the least-squares method.

\section{Results}

Preliminary experiments showed that the reactions are not sensitive to changes in the ionic strength. Hence no attempt was made to keep the ionic strength constant.
The rate laws and other experimental data for all the eleven amino acids studied. Since the results are similar, only the representative data are reproduced here.

\subsection{Stoichiometry}

The overall reaction may be represented as under.

$$
\begin{aligned}
\mathrm{RCH}\left(\mathrm{NH}_{2}\right) \mathrm{COOH} & +\mathrm{Br}_{3}^{-}+\mathrm{H}_{2} \mathrm{O} \rightarrow \mathrm{RCHO} \\
& +\mathrm{NH}_{4}^{+}+\mathrm{CO}_{2}+3 \mathrm{Br}^{-}+\mathrm{H}^{+} .
\end{aligned}
$$

\subsection{Rate laws}

The reaction is first order with respect to TBATB. The individual kinetic runs obeyed first-order kinetics. Further, the values of $k_{\mathrm{obs}}$ are independent of the initial concentration of TBATB. The order is more that one but less than two in respect of glycine (Gly), $\alpha$-alanine (Ala), 2-aminobutanoic acid (ABA), norlucine (NLE), norvaline (NVA) and phenylalanine (Phe) whereas the order with respect to valine (Val), leucine (Leu), isoleucine (Ile), aspartic acid (Asp) and glutamic acid (Glu) is two. The rate constants with respect of Ala and Leu are recorded in table 1 . Plots of $1 / k_{\text {obs }}$ against $1 /$ [amino acid] ${ }^{2}$ for Gly, Ala, ABA, NLE, NVA and Phe are linear $\left(r^{2}>\right.$ $0.995)$ with an intercept on the rate ordinate. Thus Michaelis-Menten type kinetics are observed with respect to these amino acids. This leads to the postulation of the following overall mechanism and rate law.

Table 1. Rate constants for the oxidation of oxalanine

\begin{tabular}{|c|c|c|c|c|}
\hline \multirow{2}{*}{$\frac{10^{3} \text { [TBATB] }}{\mathrm{mol} \mathrm{dm}^{-3}}$} & \multirow{2}{*}{$\frac{[\mathrm{Ala}]}{\mathrm{mol} \mathrm{dm}^{-3}}$} & \multirow{2}{*}{$\frac{[\mathrm{Leu}]}{\mathrm{mol} \mathrm{dm^{-3 }}}$} & \multicolumn{2}{|c|}{$10^{4} k_{\mathrm{obs}}\left(\mathrm{s}^{-1}\right)$} \\
\hline & & & Ala & Leu \\
\hline 1.0 & $0 \cdot 10$ & $0 \cdot 10$ & 1.87 & 1.85 \\
\hline $1 \cdot 0$ & 0.20 & 0.20 & $6 \cdot 70$ & 7.51 \\
\hline $1 \cdot 0$ & 0.40 & 0.30 & $19 \cdot 0$ & $16 \cdot 6$ \\
\hline $1 \cdot 0$ & 0.75 & 0.40 & 33.5 & $30 \cdot 1$ \\
\hline $1 \cdot 0$ & 1.00 & 0.50 & 38.9 & $46 \cdot 5$ \\
\hline $1 \cdot 0$ & 1.50 & 0.75 & $43 \cdot 3$ & 107 \\
\hline $1 \cdot 0$ & $2 \cdot 00$ & 1.00 & $46 \cdot 3$ & 190 \\
\hline $2 \cdot 0$ & 0.40 & 0.40 & 18.7 & 30.5 \\
\hline $4 \cdot 0$ & 0.40 & 0.40 & $19 \cdot 1$ & 29.5 \\
\hline $6 \cdot 0$ & 0.40 & 0.40 & 19.4 & $30 \cdot 3$ \\
\hline 8.0 & 0.40 & 0.40 & $19 \cdot 2$ & $30 \cdot 0$ \\
\hline $1 \cdot 0$ & $0 \cdot 20$ & $0 \cdot 20$ & $6 \cdot 75^{*}$ & $7.61 *$ \\
\hline
\end{tabular}
and leucine by TBATB at $308 \mathrm{~K}$.

*Contained $0.005 \mathrm{~mol} \mathrm{dm}^{-3}$ acrylonitrile 
2 Amino acid + TBATB $\stackrel{K}{\leftrightarrows}[$ intermediate $]$,

[intermediate $\stackrel{k_{2}}{\longrightarrow}$ products,

$$
\text { rate } \left.=K k_{2} \text { [amino acid }\right]^{2}[\mathrm{TBATB}] /
$$

$$
\left.(1+K \text { [amino acid }]^{2}\right) \text {. }
$$

It is proposed that the oxidation of Val, Leu, Ile, Asp and Glu also follow similar mechanism but in their case, the equilibrium constants have very small values. Thus in these cases, $K$ [amino acid] ${ }^{2} \ll 1$ and no Michaelis-Menten type kinetics is observed but a second-order dependence is obtained. The reason for this must be steric. The amino acids exhibiting second-order dependence have bulkier substituents as compared to the ones showing Michaelis-Menten kinetics. The variation in amino acid concentration was studied at different temperatures and the values of $K$ and $k_{2}$ evaluated from the double reciprocal plots. The thermodynamic and activation parameters for the formation and disproportionation of the intermediate were calculated from the values of $K$ and $k_{2}$ respectively, at different temperatures (tables 2 and 3). The reaction (2) is likely to take place in two steps. Therefore, the equilibrium constant, $K$, might be a composite quantity.

The dependence of reaction on hydrogen-ion concentration could not be determined by an addition of a mineral acid like perchloric acid as TBATB decomposed rapidly in the presence of a mineral acid. Therefore, effect of sodium acetate on the reaction rate was studied. Results showed that addition of sodium acetate has no effect on the rate of the oxidation (table 4).

\subsection{Induced polymerization of acrylonitrile}

The oxidation of amino acids, in an atmosphere of nitrogen, failed to induce the polymerization of acrylonitrile. Further, the addition of acrylonitrile had no effect on the rate of oxidation (table 1).

\begin{tabular}{|c|c|c|c|c|c|c|c|}
\hline \multirow[b]{2}{*}{ Substrate } & \multicolumn{4}{|c|}{$K\left(\mathrm{dm}^{3} \mathrm{~mol}^{-1}\right)$ at } & \multirow{2}{*}{$\frac{\Delta H}{\mathrm{~kJ} \mathrm{~mol}^{-1}}$} & \multirow{2}{*}{$\frac{\Delta S}{\mathrm{~J} \mathrm{~mol}^{-1} \mathrm{~K}^{-1}}$} & \multirow{2}{*}{$\frac{\Delta G}{\mathrm{~kJ} \mathrm{~mol}^{-1}}$} \\
\hline & $298 \mathrm{~K}$ & $308 \mathrm{~K}$ & $318 \mathrm{~K}$ & $328 \mathrm{~K}$ & & & \\
\hline $\mathrm{H}$ & $3 \cdot 20$ & $2 \cdot 72$ & $2 \cdot 21$ & 1.75 & $-18 \cdot 9 \pm 0.8$ & $-45 \pm 3$ & $-5 \cdot 4 \pm 0.6$ \\
\hline $\mathrm{Me}$ & $4 \cdot 85$ & $4 \cdot 03$ & $3 \cdot 27$ & $2 \cdot 55$ & $-19 \cdot 8 \pm 0 \cdot 8$ & $-45 \pm 2$ & $-6.5 \pm 0.6$ \\
\hline $\mathrm{PhCH}_{2}$ & $3 \cdot 97$ & $3 \cdot 34$ & $2 \cdot 72$ & $2 \cdot 16$ & $-19 \cdot 0 \pm 0 \cdot 7$ & $-44 \pm 2$ & $-6 \cdot 0 \pm 0.6$ \\
\hline $\mathrm{ABA}$ & $5 \cdot 32$ & $4 \cdot 12$ & $3 \cdot 35$ & $2 \cdot 60$ & $-21 \cdot 6 \pm 0.4$ & $-51 \pm 1$ & $-6 \cdot 6 \pm 0 \cdot 4$ \\
\hline NLE & $6 \cdot 03$ & $5 \cdot 05$ & $4 \cdot 10$ & $3 \cdot 26$ & $-19 \cdot 2 \pm 0 \cdot 7$ & $-41 \pm 2$ & $-7 \cdot 0 \pm 0.5$ \\
\hline NVA & $4 \cdot 13$ & $3 \cdot 70$ & $3 \cdot 35$ & $2 \cdot 91$ & $-12 \cdot 8 \pm 0 \cdot 6$ & $-20 \pm 2$ & $-6 \cdot 0 \pm 0.5$ \\
\hline
\end{tabular}

Table 2. Formation constants of the TBATB-amino acid complexes and the thermodynamic parameters.

\begin{tabular}{|c|c|c|c|c|c|c|c|}
\hline \multirow[b]{2}{*}{ Substrate } & \multicolumn{4}{|c|}{$10^{4} k_{2}\left(\mathrm{~s}^{-1}\right)$ at } & \multirow{2}{*}{$\frac{\Delta H^{*}}{\mathrm{~kJ} \mathrm{~mol}^{-1}}$} & \multirow{2}{*}{$\frac{\Delta S^{*}}{\mathrm{~J} \mathrm{~mol}^{-1} \mathrm{~K}^{-1}}$} & \multirow{2}{*}{$\frac{\Delta G^{*}}{\mathrm{~kJ} \mathrm{~mol}^{-1}}$} \\
\hline & $298 \mathrm{~K}$ & $308 \mathrm{~K}$ & $318 \mathrm{~K}$ & $328 \mathrm{~K}$ & & & \\
\hline Gly & $2 \cdot 23$ & $4 \cdot 81$ & $10 \cdot 6$ & $21 \cdot 3$ & $58 \cdot 9 \pm 0 \cdot 5$ & $-99 \pm 2$ & $88 \cdot 2 \pm 0 \cdot 4$ \\
\hline Ala & $27 \cdot 3$ & $48 \cdot 3$ & $86 \cdot 4$ & 148 & $43 \cdot 4 \pm 0 \cdot 4$ & $-130 \pm 1$ & $82 \cdot 0 \pm 0 \cdot 3$ \\
\hline $\mathrm{ABA}$ & $36 \cdot 8$ & $63 \cdot 9$ & 110 & 185 & $41 \cdot 3 \pm 0 \cdot 5$ & $-135 \pm 2$ & $81 \cdot 2 \pm 0.5$ \\
\hline NVA & $55 \cdot 1$ & $92 \cdot 1$ & 151 & 256 & $38.9 \pm 0.7$ & $-139 \pm 3$ & $80 \cdot 2 \pm 0 \cdot 6$ \\
\hline NLE & $59 \cdot 9$ & $97 \cdot 5$ & 164 & 271 & $38.8 \pm 0.6$ & $-139 \pm 2$ & $80 \cdot 1 \pm 0 \cdot 4$ \\
\hline Phe & $87 \cdot 5$ & 140 & 215 & 347 & $34 \cdot 5 \pm 0 \cdot 7$ & $-150 \pm 2$ & $79 \cdot 1 \pm 0 \cdot 6$ \\
\hline \multicolumn{8}{|c|}{$10^{4} k_{2}\left(\mathrm{dm}^{6} \mathrm{~mol}^{-2} \mathrm{~s}^{-1}\right)$} \\
\hline Val & $71 \cdot 7$ & 115 & 195 & 308 & $37 \cdot 3 \pm 0 \cdot 6$ & $-142 \pm 2$ & $79 \cdot 6 \pm 0.5$ \\
\hline Leu & 121 & 187 & 300 & 472 & $34 \cdot 5 \pm 0.7$ & $-148 \pm 2$ & $78 \cdot 3 \pm 0.5$ \\
\hline Ile & 180 & 285 & 440 & 671 & $33 \cdot 1 \pm 0 \cdot 6$ & $-149 \pm 2$ & $77 \cdot 3 \pm 0.6$ \\
\hline Asp & $6 \cdot 83$ & $16 \cdot 3$ & $33 \cdot 4$ & $70 \cdot 1$ & $56 \cdot 4 \pm 1 \cdot 0$ & $-97 \pm 4$ & $85 \cdot 3 \pm 1 \cdot 0$ \\
\hline Glu & $22 \cdot 2$ & $38 \cdot 7$ & $75 \cdot 2$ & 134 & $46 \cdot 7 \pm 1 \cdot 1$ & $-121 \pm 4$ & $82 \cdot 5 \pm 0 \cdot 9$ \\
\hline
\end{tabular}

Table 3. Rate of the decomposition of the intermediate complexes and the activation parameters. 
Table 4. Effect of sodium acetate on the rate of oxidation of leucine by TBATB. $[$ TBATB $]=0.001 \mathrm{~mol} \mathrm{dm}^{-3} ;[\mathrm{Leu}]=0.40 \mathrm{~mol} \mathrm{dm}^{-3} ;$ Temp. $=308 \mathrm{~K}$.

\begin{tabular}{lcccccc}
\hline$[\mathrm{NaOAc}]\left(\mathrm{mol} \mathrm{dm}^{-3}\right)$ & $0 \cdot 00$ & $0 \cdot 10$ & 0.20 & $0 \cdot 30$ & $0 \cdot 40$ & 0.50 \\
$10^{4} k_{\text {obs }}\left(\mathrm{s}^{-1}\right)$ & $30 \cdot 1$ & $29 \cdot 8$ & $30 \cdot 7$ & $29 \cdot 3$ & $31 \cdot 5$ & $30 \cdot 4$ \\
\hline
\end{tabular}

Table 5. Effect of tetrabutylammonium chloride on the oxidation of leucine by TBATB. $[$ TBATB $]=0.001 \mathrm{~mol} \mathrm{dm}^{-3} ;[\mathrm{Leu}]=0.40 \mathrm{~mol} \mathrm{dm}^{-3} ;$ Temp. $=308 \mathrm{~K}$.

\begin{tabular}{|c|c|c|c|c|c|c|}
\hline $10^{3}[\mathrm{TBACl}]\left(\mathrm{mol} \mathrm{dm}^{-3}\right)$ & $0 \cdot 00$ & $0 \cdot 5$ & $1 \cdot 0$ & $2 \cdot 0$ & $3 \cdot 0$ & $4 \cdot 0$ \\
\hline $10^{4} k_{\mathrm{obs}}\left(\mathrm{s}^{-1}\right)$ & $30 \cdot 1$ & $30 \cdot 3$ & $29 \cdot 5$ & $29 \cdot 9$ & $30 \cdot 5$ & $30 \cdot 0$ \\
\hline
\end{tabular}

Table 6. Dependence of rate of oxidation of leucine on the concentration of bromide ions. $[$ TBATB $]=0.001 \mathrm{~mol} \mathrm{dm}^{-3} ;[\mathrm{Leu}]=0.40 \mathrm{~mol} \mathrm{dm}^{-3} ;$ Temp. $=308 \mathrm{~K}$.

\begin{tabular}{lrccccccc}
\hline$\left[\mathrm{Br}^{-}\right]\left(\mathrm{mol} \mathrm{dm}^{-3}\right)$ & 0.0 & 0.04 & 0.08 & $0 \cdot 12$ & $0 \cdot 16$ & $0 \cdot 20$ & $0 \cdot 24$ & 0.30 \\
$10^{4} k_{\text {obs }}\left(\mathrm{s}^{-1}\right)$ & $30 \cdot 1$ & $26 \cdot 9$ & $22 \cdot 6$ & $19 \cdot 8$ & $16 \cdot 0$ & $15 \cdot 8$ & $15 \cdot 7$ & $15 \cdot 8$ \\
\hline
\end{tabular}

Table 7. Dependence of the rate of oxidation of leucine by TBATB on solvents compositions.

\begin{tabular}{llllll}
{$[$ TBATB $]=0.001 \mathrm{~mol} \mathrm{dm}^{-3} ;[\mathrm{Leu}]=0.40 \mathrm{~mol} \mathrm{dm}^{-3} ;$ Temp. $=308 \mathrm{~K}$.} \\
\hline$\% \mathrm{AcOH}(v / v)$ & 25 & 40 & 50 & 60 & 70 \\
$10^{4} k_{\mathrm{obs}}\left(\mathrm{s}^{-1}\right)$ & $53 \cdot 8$ & $38 \cdot 3$ & $30 \cdot 1$ & $26 \cdot 4$ & $22 \cdot 7$ \\
\hline
\end{tabular}

\subsection{Effect of tetrabutylammonium ions}

The rates of oxidation were not affected by the addition of tetrabutylammonium chloride (TBACl) (table 5).

\subsection{Effect of bromide ions}

The rate of oxidation decreases with an increase in the concentration of potassium bromide but reaches a limiting value at $[\mathrm{KBr}] \approx 0.16 \mathrm{~mol} \mathrm{dm}^{-3}$ (table 6).

\subsection{Effect of solvent composition}

The rate of oxidation was determined in solvents containing different amounts of acetic acid and water. It was observed that the rate increased with an increase in the amount of water in the solvent mixture (table 7).

\section{Discussion}

An isokinetic plot between activation enthalpies and entropies of oxidation of the eleven amino acids is reasonably good $\left(r^{2}=0.9887\right.$, s.d. $\left.=0.97\right)$. The value of isokinetic temperature is $460 \pm 16 \mathrm{~K}$. The isokinetic relationship was verified and found genuine by applying Exner's ${ }^{14}$ criterion. The Exner plot between $\log k_{2}$ at $298 \mathrm{~K}$ and $\log k_{2}$ at $328 \mathrm{~K}$ is linear $\left(r^{2}=0.9969, \quad\right.$ s.d. $=0.02 ; \quad$ slope $\left.=0.7571 \pm 0.0140\right)$. The isokinetic temperature is $496 \pm 14 \mathrm{~K}$, which is in very good agreement with the value obtained from the activation parameter data. The linear isokinetic correlation implies that all the amino acids are oxidized by the same mechanism and the changes in the rate are governed by the changes in both the enthalpy and entropy of the activation.

\subsection{Reactive oxidizing species}

We have earlier ${ }^{8}$ carried out some conductivity measurements to determine the nature of TBATB in aqueous acetic acid solution. It was observed that acetic acid has very low conductivity. Addition of BATB increases the conductivity of the solution. We measured the conductivity of TBATB in solvents containing different proportions of acetic acid (100$30 \%$ ) and water. We found that the conductivity increases sharply as the water content is increased and 
reaches a limiting value in about $60 \%$ acetic acidwater mixture. TBATB can be considered an ionic compound, which exists under our reaction conditions as tetrabutylammonium and tribromide ions as shown in (5). No effect of added tetrabutylammonium ion also indicates that the equilibrium (5) lies far towards the right. Similar results were obtained in the oxidation of aliphatic aldehydes by TBATB. ${ }^{8}$ Thus in the present reaction also the reactive oxidizing species is the tribromide ion,

$$
\left(\mathrm{C}_{4} \mathrm{H}_{9}\right)_{4} \mathrm{NBr}_{3} \leftrightarrows\left(\mathrm{C}_{4} \mathrm{H}_{9}\right)_{4} \mathrm{~N}^{+}+\mathrm{Br}_{3}^{-}
$$

Tribromide ion is known to dissociate to bromine and bromide ion and the value of the dissociation constant has been reported. ${ }^{13}$ The effect of addition of bromide ion (cf. table 6) indicated that as the $\left[\mathrm{Br}^{-}\right]$increases, the concentration of bromine and its contribution to the oxidation decrease and become almost negligible at $[\mathrm{KBr}] \approx 0.16 \mathrm{~mol} \mathrm{dm}^{-3}$. As a large excess $\left(0.3 \mathrm{~mol} \mathrm{dm}^{-3}\right)$ of bromide ion has been added in present reaction, the oxidation due to bromine will be suppressed. Thus in the present reaction the reactive oxidizing species is the tribromide ion.

$$
\mathrm{Br}_{3}^{-} \leftrightarrows \mathrm{Br}_{2}+\mathrm{Br}^{-}
$$

\subsection{Mechanism}

In aqueous solutions, amino acids are known to exist in the zwitterionic form.

$$
\mathrm{RCH}\left(\mathrm{NH}_{2}\right) \mathrm{COOH} \leftrightarrows \stackrel{+}{\mathrm{RCH}}\left(\stackrel{\mathrm{NH}_{3}}{)} \mathrm{COO}^{-}\right.
$$

The formation of zwitterions is facilitated by the increased polarity of the solvent due to better solvation of the ionic species. Therefore, the fact that the rate increases with an increase in polarity of the solvent suggests that the zwitterionic form is the reactive reducing species.

From the rate-law, it is apparent that a 1:2 intermediate complex of the amino acid and tribromide is formed in a rapid pre-equilibrium. However, with the present data, it is not possible to state definitely about the nature of the complex. An intermediate complex may be formed by the interaction between the non-bonded pairs of electrons of the carboxylicoxygen and tribromide ion. The formation of a similar complex has been postulated in the oxidation of alcohols $^{15}$ by pyridinium hydrobromide perbromide
(PHPB) also. The formation of moderately stable complexes is supported by the values of thermodynamic parameters also. The complex formation is favoured by the enthalpy term but there is a loss of entropy indicating the formation of a rigid structure. The following mechanism (scheme 1) accounts for all the observed data.

Alternatively an intermediate complex $\mathrm{C}_{1}$ may be formed between the tribromide ion and a molecule of amino acid, followed by the interaction of $\mathrm{C}_{1}$ and a molecule to yield the complex $\mathrm{C}_{2}$, which breaks down to the products in the rate-determining step.

The observed negative entropy of activation also supports a polar transition state. As the charge separation takes place, the charged ends become highly solvated. This results in an immobilization of a large number of solvent molecules, reflected in the loss of entropy.

\subsection{Correlation of structure and reactivity}

The formation constant, $K$, of the TBATB-amino acid intermediate complex does not vary much with the nature of the substituents. Similar observation has been recorded in the oxidation of alcohols by PHPB. ${ }^{15}$ However, the rate of disproportionation of the intermediate or the rate constant, as the case may be, showed wide variation. These values were therefore used for correlation analysis.

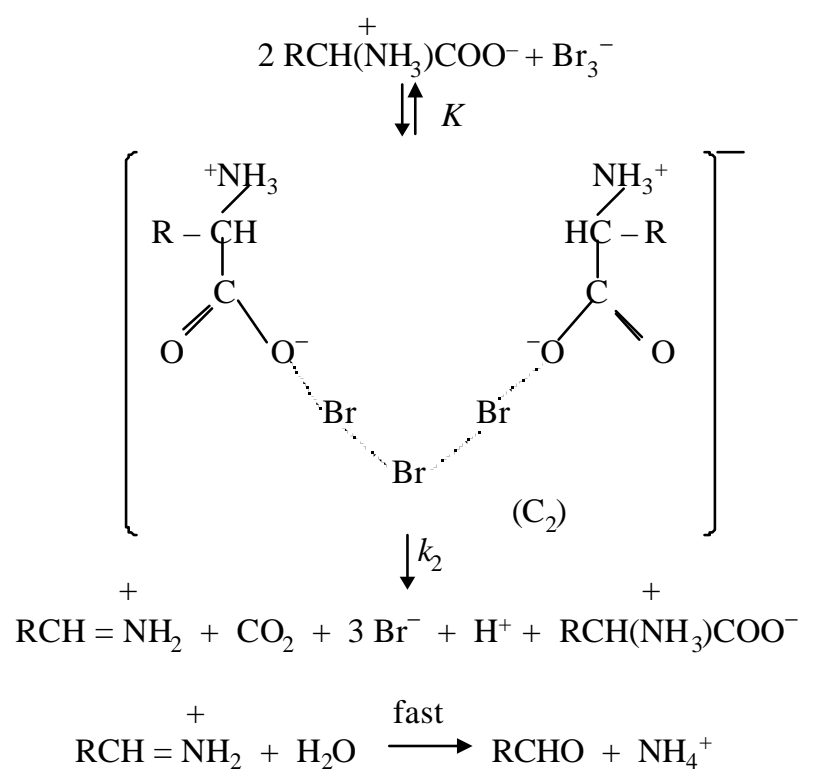

Scheme 1. 
Table 8. Temperature dependence of the reaction constants.

\begin{tabular}{lccccc}
\hline Temp.(K) & $\rho^{*}$ & $\delta$ & $R^{2}$ & s.d. & $\psi$ \\
\hline 298 & $-0.77 \pm 0.01$ & $-0.58 \pm 0.01$ & 0.9997 & 0.01 & 0.02 \\
308 & $-0.64 \pm 0.02$ & $-0.55 \pm 0.01$ & 0.9988 & 0.02 & 0.04 \\
318 & $-0.57 \pm 0.01$ & $-0.51 \pm 0.01$ & 0.9994 & 0.01 & 0.03 \\
328 & $-0.52 \pm 0.02$ & $-0.47 \pm 0.01$ & 0.9998 & 0.01 & 0.02 \\
\hline
\end{tabular}

The rate constants of the aliphatic amino acids failed to give any significant correlation with Taft's polar and steric effects separately. The rates were therefore analysed in terms of Pavelich-Taft's ${ }^{16}$ dual substituent parameter (10).

$$
\begin{gathered}
\log k_{2}=-1 \cdot 12 \pm 0.29 \sigma^{*}-2 \cdot 33, \\
r^{2}=0.6518 ; \text { s.d. }=0.36 ; \psi=0 \cdot 62 ; \\
n=10 ; T=298 \mathrm{~K} . \\
\log k_{2}=-0.77 \pm 0 \cdot 16 E_{s}-2 \cdot 70, \\
r^{2}=0.7439 ; \text { s.d. }=0.31 ; \psi=0 \cdot 53 ; \\
n=10 ; T=298 \mathrm{~K} . \\
\log k_{2}=\sigma^{*} \rho^{*}+\delta E_{s}+\log k_{0} .
\end{gathered}
$$

Here $n$ is the number of data points and $\psi$ is Exner's statistical parameter. ${ }^{17}$

The correlations in terms of (9) are excellent (table 8). The reaction constants have negative values. The analysis showed that the reaction is susceptible to both the polar and steric effects of the substituents. The values of the reaction constants support the proposed mechanism. The negative polar reaction constant is in accordance with the net flow of electrons towards the oxidant. An increase in the electron density at the reaction centre facilitates the flow of the electrons from substrate towards the oxidant. The negative steric reaction constant indicates a steric acceleration. This may well be due to high ground state energy of the more substituted amino acids. The steric crowding is relieved in the product $\left(\mathrm{RCH}=\mathrm{NH}_{2}\right)^{+}$and as well as in the transition leading to it. This coupled with the fact that there is not much difference in the transition state energy of the crowded and uncrowded molecules explain the steric acceleration.

\section{Acknowledgement}

Thanks are due to Department of Science \& Technology, Govt. of India for financial support.

\section{References}

1. Kajigaeshi S, Kakinami T, Okamoto $\mathrm{T}$ and Fujisaki $\mathrm{S}$ 1987 Bull. Chem. Soc. Jpn. 601159

2. Kajigaeshi S, Kakinami T, Yamasaki H, Fujisaki S and Okamoto T 1988 Bull. Chem. Soc. Jpn. 612681

3. Buckles R E, Popov A I, Zelenzy W F and Smith R J $1951 \mathrm{~J}$. Am. Chem. Soc. 734525

4. Choudhary M K, Khan A T and Patel B K 1998 Tetrahedron Lett. 398163

5. Berthalot J, Guette C, Ouchefoune M, Desbens P L and Basselier J J 1986a J. Chem. Res. (S), 381; Berthalot J, Guette C, Ouchefoune M, Desbens P L and Basselier J J 1986b Synth. Commun. 161641

6. Kajigaeshi S, Kawamuki H and Fujisaki S 1989 Bull. Chem. Soc. Jpn. 622585

7. Kajigaeshi S, Morikawa Y, Fujisaki S, Kakinami T and Nishihira K 1991 Bull. Chem. Soc. Jpn. 64336

8. Baghmar M and Sharma PK 2001 Int. J. Chem. Kinet. 33390

9. Baghmar M and Sharma PK 2001 Proc. Indian Acad. Sci. (Chem. Sci.) 113139

10. Kumar A, Choudhary K, Sharma P K and Banerji K K 2001 Indian J. Chem. A40 252

11. Perrin D D, Armarego W L and Perrin D R 1966 Purification of organic compounds (Oxford: Pergamon)

12. Feigl F 1954 Spot tests (Amsterdam: Elsevier) p. 20

13. Bradfield A E, Jones B and Orton K J P 1929 J. Chem. Soc. 2810

14. Exner O 1973 Prog. Phys. Org. Chem. 10411

15. Mathur D, Sharma P K and Banerji K K 1993 J. Chem. Soc., Perkin Trans. 2205

16. Pavelich W A and Taft R W 1957 J. Am. Chem. Soc. 794935

17. Exner O 1964 Collect. Czech. Chem. Commun. 29 1094 